\title{
A combinatorial approach to partitions with parts in the gaps
}

\author{
by
}

\author{
Dennis EichHoRn (Urbana, Ill.)
}

Many links exist between ordinary partitions and partitions with parts in the "gaps". In this paper, we explore combinatorial explanations for some of these links, along with some natural generalizations. In particular, if we let $p_{k, m}(j, n)$ be the number of partitions of $n$ into $j$ parts where each part is $\equiv k(\bmod m), 1 \leq k \leq m$, and we let $p_{k, m}^{*}(j, n)$ be the number of partitions of $n$ into $j$ parts where each part is $\equiv k(\bmod m)$ with parts of size $k$ in the gaps, then $p_{k, m}^{*}(j, n)=p_{k, m}(j, n)$.

1. Introduction. Recently, several papers have been written that study partitions by considering, manipulating, and even filling their "gaps". The pathbreaking work in this area has been done largely by Alladi and Bowman (see [1-4]). In this paper, we explore how taking a combinatorial approach to this gap-theoretic study of partitions can make several generalizations of results existing in the literature come to light in a natural way.

2. Preliminaries. In this section, we give the definitions necessary to state our main results. Although the symbolic representation of the objects defined in this section may seem lengthy, the objects themselves are actually quite simple.

A composition of a non-negative integer $n$ is any sequence of positive integers with $\operatorname{sum} n$. An ordinary partition of a non-negative integer $n$ is a non-increasing sequence of positive integers with sum $n$. Let $\mathfrak{C}$ be the set of all compositions, let $\mathfrak{C}(n)$ be the set of all compositions of $n$, let $\mathfrak{P}$ be the set of all ordinary partitions, let $\mathfrak{P}(n)$ be the set of all ordinary partitions of $n$, and let $p(n)=|\mathfrak{P}(n)|$, the cardinality of $\mathfrak{P}(n)$. As a convention, we include $\emptyset$ in $\mathfrak{P}$ and $\mathfrak{C}$.

1991 Mathematics Subject Classification: Primary 05A17; Secondary 05A19. 
Notation. 1. For $d, e$ integers with $e \geq 1$, we define the formal sum:

$$
d^{e}=\underbrace{d+\ldots+d}_{e \text { times }} \text {. }
$$

2. For $d_{i}$ integers, we denote the composition $d_{a}^{e_{a}}+d_{a+1}^{e_{a+1}}+\ldots+d_{b}^{e_{b}}$ by $\bigoplus_{i=a}^{b} d_{i}^{e_{i}}$.

3 . When there is a risk of confusion, we let $\oplus$ denote the concatenation of two pieces of a composition or partition, and we let + denote the sum within a part of a composition or partition (that is, $\oplus$ is a binary operation from $\mathfrak{C}(n) \times \mathfrak{C}(m)$ into $\mathfrak{C}(n+m)$ while + is a binary operation from $\mathbb{Z} \times \mathbb{Z}$ into $\mathbb{Z})$.

\section{EXAMPLES.}

- $14+13+10+10+7+7+7+7+6+5+3+3+3 \in \mathfrak{P}(95) \subset \mathfrak{C}(95)$, and may be rewritten as

$$
\begin{gathered}
14^{1}+13^{1}+10^{2}+7^{4}+6^{1}+5^{1}+3^{3} . \\
\text { - } \bigoplus_{i=1}^{4}\left(19-i^{2}\right)^{i}=18^{1}+15^{2}+10^{3}+3^{4} \in \mathfrak{P}(90) \subset \mathfrak{C}(90) \\
=18+15+15+10+10+10+3+3+3+3 . \\
\text { - }(5+4) \oplus 6 \oplus(21+7+2) \oplus 3 \in \mathfrak{C}(48) \text { is merely } \\
9+6+30+3 .
\end{gathered}
$$

Now we are ready to define a "partition with ones in the gaps". The idea here is that we take a partition with differences at least two between consecutive parts, and we insert up to $g_{i}$ ones between the $i$ th and $(i+1)$ st parts where $g_{i}$, the "ith gapspace", is the amount of extra room we have in meeting the minimal difference two condition.

Definition. A partition with ones in the gaps is any composition that may be constructed in the following way:

- For $b \geq 0$, let $\bigoplus_{i=1}^{b} d_{i} \in \mathfrak{P}$ be such that $d_{b} \geq 2$ and $d_{i}-d_{i+1} \geq 2$ for all $1 \leq i<b$.

- Define $g_{0}=\infty, g_{b}=d_{b}-2$, and $g_{i}=d_{i}-d_{i+1}-2$ for all $1 \leq i<b$.

- Then

$$
\pi=1^{e_{0}} \oplus \bigoplus_{i=1}^{b}\left(d_{i} \oplus 1^{e_{i}}\right)
$$

where $0 \leq e_{i} \leq g_{i}$ for $i=0,1, \ldots, b$ is a partition with ones in the gaps. We call the $g_{i}$ the gapspaces of $\pi$, we call the $d_{i}$ the main parts of $\pi$, and we call the parts of size one the parts in the gaps of $\pi$. 
Let $\mathfrak{P}^{*}$ be the set of all partitions with ones in the gaps, let $\mathfrak{P}^{*}(n)$ be the set of all partitions with ones in the gaps such that the sum of the parts (including the ones) is $n$, and let $p^{*}(n)=\left|\mathfrak{P}^{*}(n)\right|$. Notice that for every $\pi \in \mathfrak{P}^{*}$, the representation of $\pi$ in (1) is unique.

\section{EXAMPLES.}

- $1^{2}+21+19+1+16+1^{2}+8+2 \in \mathfrak{P}^{*}(71) \subset \mathfrak{C}(71)$. In this example, $g_{0}=\infty, g_{1}=0, g_{2}=1, g_{3}=6, g_{4}=4$, and $g_{5}=0$.

- $30+26+19+1^{3}+14+12+1^{5}+3+1 \in \mathfrak{P}^{*}(113) \subset \mathfrak{C}(113)$. In this example, $g_{0}=\infty, g_{1}=2, g_{2}=5, g_{3}=3, g_{4}=0, g_{5}=7$, and $g_{6}=1$.

- $p^{*}(4)=5$ since $\mathfrak{P}^{*}(4)=\left\{4,3+1,1+3,1^{2}+2,1^{4}\right\} \subset \mathfrak{C}(4)$.

Let $\mathfrak{C}(j, n)$ be the set of all compositions of $n$ with $j$ parts, let $\mathfrak{P}(j, n)$ be the set of all ordinary partitions of $n$ into $j$ parts, let $p(j, n)=|\mathfrak{P}(j, n)|$, let $\mathfrak{P}^{*}(j, n)$ be the set of all partitions of $n$ into $j$ parts with ones in the gaps, and let $p^{*}(j, n)=\left|\mathfrak{P}^{*}(j, n)\right|$. (Note: when we say that $\pi \in \mathfrak{P}^{*}(j, n)$, we mean that if we write $\pi$ as in (1), then $n=e_{0}+\sum_{i=1}^{b}\left(d_{i}+e_{i}\right)$ and $j=b+\sum_{i=0}^{b} e_{i}$.)

EXAMPLES.

- $19+13^{2}+9+8+7^{3}+2 \in \mathfrak{P}(9,85) \subset \mathfrak{C}(9,85)$.

- $p(3,10)=8$ since

$$
\begin{gathered}
\mathfrak{P}(3,10)=\left\{8+1^{2}, 7+2+1,6+3+1,6+2+2,5+4+1,\right. \\
5+3+2,4+4+2,4+3+3\} \subset \mathfrak{C}(3,10) . \\
\text { - } 1^{3}+20+1^{3}+12+10+6+1^{2} \in \mathfrak{P}^{*}(12,56) \subset \mathfrak{C}(12,56) . \\
\text { - } p^{*}(3,10)=8 \text { since } \\
\mathfrak{P}^{*}(3,10)=\left\{1^{2}+8,1+8+1,8+1^{2}, 1+7+2,7+1+2,\right. \\
1+6+3,6+1+3,6+3+1\} \subset \mathfrak{C}(3,10) .
\end{gathered}
$$

Let $\mathfrak{P}_{k, m}$ be the set of all partitions where each part is $\equiv k(\bmod m)$, let $\mathfrak{P}_{k, m}(n)$ be the set of all partitions of $n$ where each part is $\equiv k(\bmod m)$, let $p_{k, m}(n)=\left|\mathfrak{P}_{k, m}(n)\right|$, let $\mathfrak{P}_{k, m}(j, n)$ be the set of all partitions of $n$ into $j$ parts where each part is $\equiv k(\bmod m)$, and let $p_{k, m}(j, n)=\left|\mathfrak{P}_{k, m}(j, n)\right|$.

We are now prepared to define a "partition where each part is $\equiv k$ $(\bmod m)$ with parts of size $k$ in the gaps". The idea here is analogous to the idea behind a "partition with ones in the gaps", but now the minimal difference two condition is replaced by a minimal difference $2 m$ condition.

Definition. Let $1 \leq k \leq m$. A partition where each part is $\equiv k$ $(\bmod m)$ with parts of size $k$ in the gaps is any composition that may be constructed in the following way:

- For $b \geq 0$, let $\bigoplus_{i=1}^{b} d_{i} \in \mathfrak{P}_{k, m}$ be such that $d_{b} \geq m+k$ and $d_{i}-d_{i+1} \geq$ $2 m$ for all $1 \leq i<b$. 
- Define $g_{0}=\infty, g_{b}=\left(d_{b}-k\right) / m-1$, and $g_{i}=\left(d_{i}-d_{i+1}\right) / m-2$ for all $1 \leq i<b$.

- Then

$$
\pi=k^{e_{0}} \oplus \bigoplus_{i=1}^{b}\left(d_{i} \oplus k^{e_{i}}\right)
$$

where $0 \leq e_{i} \leq g_{i}$ for $i=0,1, \ldots, b$ is a partition where each part is $\equiv k$ $(\bmod m)$ with parts of size $k$ in the gaps. We again call the $g_{i}$ the gapspaces of $\pi$, the $d_{i}$ the main parts of $\pi$, and the parts of size $k$ the parts in the gaps of $\pi$.

Let $\mathfrak{P}_{k, m}^{*}$ be the set of all partitions where each part is $\equiv k(\bmod m)$ with parts of size $k$ in the gaps, let $\mathfrak{P}_{k, m}^{*}(n)$ be the set of all partitions in $\mathfrak{P}_{k, m}^{*}$ such that the sum of the parts (including the parts of size $k$ ) is $n$, and let $p_{k, m}^{*}(n)=\left|\mathfrak{P}_{k, m}^{*}(n)\right|$. Notice that for every $\pi \in \mathfrak{P}_{k, m}^{*}$, the representation of $\pi$ in (2) is unique. Let $\mathfrak{P}_{k, m}^{*}(j, n)$ be the set of all partitions in $\mathfrak{P}_{k, m}^{*}(n)$ with $j$ parts (including the parts of size $k$ ), and let $p_{k, m}^{*}(j, n)=\left|\mathfrak{P}_{k, m}^{*}(j, n)\right|$. (Note: when we say that $\pi \in \mathfrak{P}_{k, m}^{*}(j, n)$, we mean that if we write $\pi$ as in (2), then $n=k \cdot e_{0}+\sum_{i=1}^{b}\left(d_{i}+k \cdot e_{i}\right)$ and $j=b+\sum_{i=0}^{b} e_{i}$.)

EXAMPLES.

- $3^{6}+79+3^{3}+59+51+23+3+11 \in \mathfrak{P}_{3,4}^{*}(15,253) \subset \mathfrak{P}_{3,4}^{*}(253)$. In this example, $g_{0}=\infty, g_{1}=3, g_{2}=0, g_{3}=5, g_{4}=1$, and $g_{5}=1$.

- $p_{1,2}^{*}(8)=6$ since

$$
\mathfrak{P}_{1,2}^{*}(8)=\left\{1+7,7+1,1^{3}+5,1^{2}+5+1,1^{5}+3,1^{8}\right\} .
$$

- $p_{2,3}^{*}(4,20)=5$ since

$$
\mathfrak{P}_{2,3}^{*}(4,20)=\left\{2^{3}+14,2^{2}+14+2,2+14+2^{2}, 14+2^{3}, 2^{2}+11+5\right\} \text {. }
$$

3. The main results. In this section, we give generalizations of some theorems of Bowman [4]. Bowman's results were originally proved using continued fraction generating functions, but here we will use purely combinatorial methods.

Theorem 1 is an essential extension of one of Bowman's results, and it allows us to later achieve further generalizations.

Theorem 1. Let $j$ and $n$ be integers. Then

$$
p^{*}(j, n)=p(j, n) .
$$

Proof. We will construct a map $\phi$ from $\mathfrak{P}^{*}(j, n)$ to $\mathfrak{P}(j, n)$, and then we will demonstrate that $\phi$ is a bijection. We can think of the action of $\phi$ on $\pi \in \mathfrak{P}^{*}(j, n)$ as a repeated stealing from the rich and giving to the poor. Each of the main parts of $\pi$ will get a turn at being "the rich", and every 
part, main or in the gaps, lying anywhere to the right of a main part will get some number of turns at being one of "the poor".

Let $\pi$ be defined as in (1). Then the action of $\phi$ on $\pi$ will be comprised of $b$ instances of stealing from the rich and giving to the poor, along with one rearrangement of parts. We will define $\phi_{t}$ to be the map that causes the $t$ th instance of stealing from the rich and giving to the poor for $t=1, \ldots, b$, and we will define $\psi$ to be the map that rearranges the parts appropriately.

Let $\Pi_{t}(j, n) \subset \mathfrak{C}(j, n)$ be the set of all $\pi_{t} \in \mathfrak{C}(j, n)$ that may be written in the following form:

$$
\pi_{t}=1^{e_{0}} \oplus \bigoplus_{i=1}^{t-1}\left[d_{i} \oplus(i+1)^{e_{i}}\right] \oplus \bigoplus_{i=t}^{b}\left[d_{i} \oplus t^{e_{i}}\right] .
$$

When we write an element of $\Pi_{t}(j, n)$ in this manner, we will refer to the $d_{i}$ as the main parts, and we will refer to all of the other parts as parts in the gaps. Let $\phi_{t}$ act on $\pi_{t} \in \Pi_{t}(j, n)$ by "stealing" the quantity $b-t+\sum_{i=t}^{b} e_{i}$ from $d_{t}$ and "giving" one to each part of $\pi_{t}$ that lies to the right of $d_{t}$. We can see that $\phi_{t}$ is actually a map from $\Pi_{t}(j, n)$ into $\Pi_{t+1}(j, n)$. Let $\psi$ reorder the parts of $\pi_{b+1} \in \Pi_{b+1}(j, n)$ by first taking all of the main parts of $\pi_{b+1}$ in order and then appending all of the parts in the gaps of $\pi_{b+1}$ in reverse order. Given these definitions of $\psi$ and the $\phi_{t}$, we let $\phi$ be defined by $\phi(\pi)=\psi \circ \phi_{b} \circ \phi_{b-1} \circ \ldots \circ \phi_{1}(\pi)$ where $\pi \in \mathfrak{P}^{*}(j, n)$ is written as in (1).

Example. Consider $\pi_{1} \in \mathfrak{P}^{*}(13,80)$ defined as follows:

$$
\pi_{1}=1^{3}+23+1+19+17+10+1^{4}+3 .
$$

Notice that $\pi_{1} \in \Pi_{1}(13,80)$, and thus

$$
\begin{aligned}
& \phi_{1}\left(\pi_{1}\right)=1^{3}+14+2+20+18+11+2^{4}+4=\pi_{2}, \\
& \phi_{2}\left(\pi_{2}\right)=1^{3}+14+2+13+19+12+3^{4}+5=\pi_{3}, \\
& \phi_{3}\left(\pi_{3}\right)=1^{3}+14+2+13+13+13+4^{4}+6=\pi_{4}, \\
& \phi_{4}\left(\pi_{4}\right)=1^{3}+14+2+13+13+8+5^{4}+7=\pi_{5}, \\
& \phi_{5}\left(\pi_{5}\right)=1^{3}+14+2+13+13+8+5^{4}+7=\pi_{6},
\end{aligned}
$$

and

$$
\psi\left(\pi_{6}\right)=14+13^{2}+8+7+5^{4}+2+1^{3}=\phi\left(\pi_{1}\right) .
$$

Symbolically, we have defined $\phi_{t}$ by

$$
\begin{array}{r}
\phi_{t}\left(\pi_{t}\right)=1^{e_{0}} \oplus \bigoplus_{i=1}^{t-1}\left[d_{i} \oplus(i+1)^{e_{i}}\right] \oplus\left(d_{t}-b+t-\sum_{r=t}^{b} e_{r}\right) \oplus \ldots \\
\ldots \oplus(t+1)^{e_{t}} \oplus \bigoplus_{i=t+1}^{b}\left[d_{i}+1 \oplus(t+1)^{e_{i}}\right]
\end{array}
$$


where for $t=1, \ldots, b, \pi_{t} \in \Pi_{t}(j, n)$ is defined as in (3). (Note: when we write $\pi_{t+1}=\phi_{t}\left(\pi_{t}\right)$ as in $(3)$, the $d_{i}$ are not the same as the $d_{i}$ from $\pi_{t}$.) We can also see that we have defined $\psi$ by

$$
\psi\left(\pi_{b+1}\right)=\left[\bigoplus_{i=1}^{b} d_{i}\right] \oplus\left[\bigoplus_{i=0}^{b}(1+b-i)^{e_{b-i}}\right]
$$

where $\pi_{b+1} \in \Pi_{b+1}(j, n)$ is defined as in (3). We find that the action of $\phi$ on $\pi$ is given symbolically by

$$
\begin{aligned}
\phi(\pi) & =\left[\bigoplus_{i=1}^{b}\left(d_{i}-j+2 i-1+\sum_{t=0}^{i-1} e_{t}\right)\right] \oplus\left[\bigoplus_{i=0}^{b}(1+b-i)^{e_{b-i}}\right] \\
& =\bigoplus_{i=1}^{j} a_{i} \in \mathfrak{C}(j, n),
\end{aligned}
$$

where again, $\pi$ is written as in (1).

With these symbolic definitions in place, we are now prepared to show that $\phi$ is actually a map from $\mathfrak{P}^{*}(j, n)$ into $\mathfrak{P}(j, n)$, and also that $\phi$ is a bijection. Notice that, for $1 \leq i<b$,

$$
\begin{aligned}
a_{i} & =d_{i}-j+2 i-1+\sum_{t=0}^{i-1} e_{t} \\
& =d_{i}-2-j+2(i+1)-1-e_{i}+\sum_{t=0}^{(i+1)-1} e_{t} \\
& =a_{i+1}+d_{i}-d_{i+1}-2-e_{i}=a_{i+1}+g_{i}-e_{i} \geq a_{i+1} .
\end{aligned}
$$

Also notice that

$$
\begin{aligned}
a_{b} & =d_{b}-j+2 b-1+\sum_{t=0}^{b-1} e_{t}=d_{b}-2-e_{b}+b+1 \\
& =g_{b}-e_{b}+b+1 \geq b+1 \geq a_{b+1} .
\end{aligned}
$$

Furthermore, notice from (4) that for $b<i \leq j$, the $a_{i}$ are non-increasing, and thus $\phi(\pi) \in \mathfrak{P}(j, n)$.

Next, suppose

$$
\phi\left[1^{e_{0}} \oplus \bigoplus_{i=1}^{b}\left(d_{i} \oplus 1^{e_{i}}\right)\right]=\beta=\bigoplus_{i=1}^{j} a_{i} \in \mathfrak{P}(j, n) .
$$

We now show that $\phi$ is one-to-one by demonstrating that $b$, the $e_{i}$, and the $d_{i}$ are all uniquely determined by $\beta$. First, to see that $b$ is uniquely determined, we observe that by (4), $a_{b+1}=h+1$ where $h$ is the largest integer such that $e_{h} \neq 0$ (if there is no such $h$, then $b=j$ ), and thus $a_{b+1} \leq b+1$. Now, since 
$a_{b} \geq b+1$ by (5), we find that $b$ must be the smallest integer such that $a_{b+1} \leq b+1$, and thus $b$ is uniquely determined by $\beta$. Next, we can see from (4) that each $e_{i}$ is exactly the multiplicity of the parts of size $i+1$ in $\beta$ for each $i=0,1, \ldots, b-1$, and thus these $e_{i}$ are also uniquely determined by $\beta$. Given that, $e_{b}=j-b-\sum_{t=0}^{b-1} e_{t}$ is also uniquely determined by $\beta$. Finally, (4) tells us that $d_{i}=a_{i}+j-2 i+1-\sum_{t=0}^{i-1} e_{t}$ for all $1 \leq i \leq b$. Therefore, each part of $1^{e_{0}} \oplus \bigoplus_{i=1}^{b}\left(d_{i} \oplus 1^{e_{i}}\right)$ is uniquely determined by $\beta$, and thus $\phi$ is one-to-one.

Now we show that $\phi$, as a map from $\mathfrak{P}^{*}(j, n)$ into $\mathfrak{P}(j, n)$, is onto. Suppose $\gamma=\bigoplus_{i=1}^{j} a_{i} \in \mathfrak{P}(j, n)$. Let $b$ be the smallest positive integer such that $a_{b+1} \leq b+1$. For $0 \leq t \leq b-1$, let $e_{t}$ be the number of $a_{i}=t+1$. Let $e_{b}=j-b-\sum_{i=1}^{b-1} e_{i}$. For $1 \leq i \leq b$, let $d_{i}=a_{i}+j-2 i+1-\sum_{t=0}^{i-1} e_{t}$. Consider

$$
\phi\left[1^{e_{0}} \oplus \bigoplus_{i=1}^{b}\left(d_{i} \oplus 1^{e_{i}}\right)\right]=\bigoplus_{i=1}^{j} c_{i} \in \mathfrak{P}(j, n) .
$$

By (4), we can see that both for $1 \leq i \leq b$ and for $b<i \leq j, c_{i}=a_{i}$. Thus

$$
\phi\left[1^{e_{0}} \oplus \bigoplus_{i=1}^{b}\left(d_{i} \oplus 1^{e_{i}}\right)\right]=\gamma,
$$

and since $\gamma \in \mathfrak{P}(j, n)$ was arbitrary, $\phi$ is onto.

EXAMPLE. Consider $\gamma \in \mathfrak{P}(8,45)$ defined as follows:

$$
\gamma=9+9+7+6+5+5+3+1 .
$$

Since $a_{4}=6 \not \leq 4$ and $a_{5}=5 \leq 5, b=5-1=4$. Since $\gamma$ contains one 1 , no $2 \mathrm{~s}$, one 3 , and no $4 \mathrm{~s}$, we have $e_{0}=1, e_{1}=0, e_{2}=1$, and $e_{3}=0$. Also $e_{4}=8-4-1-0-1=2$. Finally, $d_{1}=9+8-2+1-1=15, d_{2}=$ $9+8-4+1-(1+0)=13, d_{3}=7+8-6+1-(1+0+1)=8$, and $d_{4}=6+8-8+1-(1+0+1+0)=5$. By $(4)$, we can now see that in fact

$$
\phi\left(1+15+13+1+8+5+1^{2}\right)=\gamma .
$$

Since $\phi$ is one-to-one and onto, it is a bijection, and hence $p^{*}(j, n)=$ $p(j, n)$.

Now, as a corollary to Theorem 1, we have a new proof of one of Bowman's results [4, Theorem 1$]$.

Corollary 1 (Bowman). Let $n$ be any integer. Then

$$
p^{*}(n)=p(n) .
$$


Proof. By Theorem 1,

$$
p^{*}(n)=\sum_{j=0}^{n} p^{*}(j, n)=\sum_{j=0}^{n} p(j, n)=p(n) .
$$

Now that we have made the key observation, we can quickly achieve our most general main theorem. This theorem is a three-fold generalization of Corollary 1.

THEOREM 2. For all non-negative integers $j, k, m$, and $n$ with $1 \leq k \leq m$,

$$
p_{k, m}^{*}(j, n)=p_{k, m}(j, n)
$$

Pr o of. We will show that there is a one-to-one correspondence between elements of $\mathfrak{P}_{k, m}^{*}(j, n)$ and elements of $\mathfrak{P}^{*}(j,(n+j(m-k)) / m)$, and then we will show that there is a one-to-one correspondence between elements of $\mathfrak{P}_{k, m}(j, n)$ and elements of $\mathfrak{P}(j,(n+j(m-k)) / m)$. After we have demonstrated this, our result will follow immediately from Theorem 1.

Recall that every $\lambda \in \mathfrak{P}_{k, m}^{*}(j, n)$ may be written as

$$
\lambda=k^{e_{0}} \oplus \bigoplus_{i=1}^{b}\left(f_{i} \oplus k^{e_{i}}\right)
$$

where $\bigoplus_{i=1}^{b} f_{i} \in \mathfrak{P}_{k, m}, f_{b} \geq m+k, f_{i}-f_{i+1} \geq 2 m$ for all $1 \leq i<b, j=$ $b+\sum_{i=0}^{b} e_{i}$, and $0 \leq e_{i} \leq g_{i}$ for $0 \leq i \leq b$ where $g_{0}=\infty, g_{b}=\left(f_{b}-k\right) / m-1$, and $g_{i}=\left(f_{i}-f_{i+1}\right) / m-2$ for all $1 \leq i<b$. By setting $d_{i}=\left(f_{i}-k\right) / m+1$, we see that this is equivalent to saying that every element of $\mathfrak{P}_{k, m}^{*}(j, n)$ may be written as

$$
\lambda=k^{e_{0}} \oplus \bigoplus_{i=1}^{b}\left\{\left[k+m\left(d_{i}-1\right)\right] \oplus k^{e_{i}}\right\}
$$

where $\bigoplus_{i=1}^{b} d_{i} \in \mathfrak{P}, d_{b} \geq 2, d_{i}-d_{i+1} \geq 2$ for all $1 \leq i<b, j=b+\sum_{i=0}^{b} e_{i}$, and $0 \leq e_{i} \leq g_{i}$ for $0 \leq i \leq b$ where $g_{0}=\infty, g_{b}=d_{b}-2$, and $g_{i}=d_{i}-d_{i+1}-2$ for all $1 \leq i<b$. Now, if we observe that

$$
e_{0}+\sum_{i=1}^{b}\left(d_{i}+e_{i}\right)=\frac{n+j(m-k)}{m},
$$

we see that the elements of $\mathfrak{P}_{k, m}^{*}(j, n)$ are in one-to-one correspondence with the elements of $\mathfrak{P}^{*}(j,(n+j(m-k)) / m)$, and thus

$$
p_{k, m}^{*}(j, n)=p^{*}\left(j, \frac{n+j(m-k)}{m}\right) .
$$

Now, observe that every element of $\mathfrak{P}_{k, m}(j, n)$ may be written as $\bigoplus_{i=1}^{j} g_{i}$ where $g_{i} \equiv k(\bmod m)$ for all $1 \leq i \leq j$. By setting $a_{i}=\left(g_{i}-k\right) / m+1$, we see that this is equivalent to saying that every element of $\mathfrak{P}_{k, m}(j, n)$ may 
be written as $\bigoplus_{i=1}^{j}\left[k+m\left(a_{i}-1\right)\right]$ where $\bigoplus_{i=1}^{j} a_{i} \in \mathfrak{P}$. Now, if we observe that

$$
\sum_{i=1}^{j} a_{i}=\frac{n+j(m-k)}{m}
$$

we see that the elements of $\mathfrak{P}_{k, m}(j, n)$ are in one-to-one correspondence with the elements of $\mathfrak{P}(j,(n+j(m-k)) / m)$, and thus

$$
p_{k, m}(j, n)=p\left(j, \frac{n+j(m-k)}{m}\right) .
$$

Finally, Theorem 1 tells us that

$$
p^{*}\left(j, \frac{n+j(m-k)}{m}\right)=p\left(j, \frac{n+j(m-k)}{m}\right),
$$

and hence Theorem 2 follows.

Now, as corollaries to Theorem 2, we have another new result and a new proof of a result of Bowman [4, Theorem 2].

COROLlaRY 2. For all non-negative integers $k, m$, and $n$ with $1 \leq k \leq m$,

$$
p_{k, m}^{*}(n)=p_{k, m}(n) .
$$

Proof. By Theorem 2,

$$
p_{k, m}^{*}(n)=\sum_{j=0}^{n} p_{k, m}^{*}(j, n)=\sum_{j=0}^{n} p_{k, m}(j, n)=p_{k, m}(n) .
$$

Corollary 3 (Bowman). For all non-negative integers $n$, if we let $q(n)$ be the number of partitions of $n$ into distinct parts, then

$$
q(n)=p_{1,2}^{*}(n) \text {. }
$$

Proof. Euler showed that $q(n)=p_{1,2}(n)$ [5]. Thus, with the aid of Corollary 2 where $k=1$ and $m=2$, we see that

$$
q(n)=p_{1,2}(n)=p_{1,2}^{*}(n) .
$$

4. Further generalizations. Bijections similar to the one employed in Theorem 1 can be used to prove many other theorems involving partitions with parts in the gaps. In this section, we list several such theorems along with some examples. We will again need to define several objects with seemingly complicated symbolic representations, but as before, the objects themselves are actually quite simple.

If we unravel what was done in the proof of Theorem 2, we can see that what we have done just amounts to changing the quantity that we steal from the rich and give to the poor. In Theorem 1, at each stage we stole one for each of the poor, while in Theorem 2 , at each stage we stole $m$ for 
each of the poor. With this in mind, it is reasonable to expect that we can further generalize Theorem 2 to treat partitions whose parts are restricted to certain residue classes modulo $m$.

Let $K=\left\{k_{1}, \ldots, k_{t}\right\}$ where $1 \leq k_{1}<\ldots<k_{t} \leq m$, and let $\mathfrak{P}_{K, m}$ be the set of all partitions such that for every part $d$ of the partition, there exists some $k \in K$ such that $d \equiv k(\bmod m)$. Let $\mathfrak{P}_{K, m}(n)$ be the set of all partitions in $\mathfrak{P}_{K, m}$ such that the sum of the parts is $n$, let $p_{K, m}(n)=$ $\left|\mathfrak{P}_{K, m}(n)\right|$, let $\mathfrak{P}_{K, m}(j, n)$ be the set of all partitions in $\mathfrak{P}_{K, m}(n)$ with $j$ parts, and let $p_{K, m}(j, n)=\left|\mathfrak{P}_{K, m}(j, n)\right|$.

Definition. Let $K=\left\{k_{1}, \ldots, k_{t}\right\}$ where $1 \leq k_{1}<\ldots<k_{t} \leq m$. A partition with congruence set $K(\bmod m)$ with parts in the gaps is any composition that may be constructed in the following way:

- For $b \geq 0$, let $\bigoplus_{i=1}^{b} d_{i} \in \mathfrak{P}_{K, m}$ be such that $d_{b} \geq m+k_{1}$ and $d_{i}-d_{i+1} \geq$ $2 m$ for all $1 \leq i<b$.

- Define $g_{0}=\infty, g_{b}=\left\lfloor\left(d_{b}-k_{1}\right) / m\right\rfloor-1$, and $g_{i}=\left\lfloor\left(d_{i}-d_{i+1}\right) / m\right\rfloor-2$ for all $1 \leq i<b$.

- Then

$$
\pi=\bigoplus_{r=1}^{t} k_{r}^{e_{r, 0}} \oplus \bigoplus_{i=1}^{b}\left(d_{i} \bigoplus_{r=1}^{t} k_{r}^{e_{r, i}}\right),
$$

where $0 \leq \sum_{r=1}^{t} e_{r, i} \leq g_{i}$ for $i=0,1, \ldots, b$ and $e_{r, b}=0$ if $d_{b}-m \sum_{r=1}^{t} e_{r, b}<$ $m+k_{r}$, is a partition with congruence set $K(\bmod m)$ with parts in the gaps.

Let $\mathfrak{P}_{K, m}^{*}$ be the set of all partitions with congruence set $K(\bmod m)$ with parts in the gaps, let $\mathfrak{P}_{K, m}^{*}(n)$ be the set of all partitions in $\mathfrak{P}_{K, m}^{*}$ such that the sum of the parts is $n$, and let $p_{K, m}^{*}(n)=\left|\mathfrak{P}_{K, m}^{*}(n)\right|$. Let $\mathfrak{P}_{K, m}^{*}(j, n)$ be the set of all partitions in $\mathfrak{P}_{K, m}^{*}(n)$ with $j$ parts, and let $p_{K, m}^{*}(j, n)=\left|\mathfrak{P}_{K, m}^{*}(j, n)\right|$.

Using the bijection from Theorem 1 with the amount we steal from the rich and give to the poor increased by a factor of $m$, we can prove

TheOREm 3. Let $j, m$, and $n$ be non-negative integers, and let $K=$ $\left\{k_{1}, \ldots, k_{t}\right\}$ where $1 \leq k_{1}<\ldots<k_{t} \leq m$. Then

$$
p_{K, m}^{*}(j, n)=p_{K, m}(j, n) .
$$

As before, summing on $j$ yields a meaningful corollary.

Corollary 4. Let $m$ and $n$ be non-negative integers, and let $K=$ $\left\{k_{1}, \ldots, k_{t}\right\}$ where $1 \leq k_{1}<\ldots<k_{t} \leq m$. Then

$$
p_{K, m}^{*}(n)=p_{K, m}(n) .
$$

ExAmple. If we let $K=\{1,2\}$, then

$$
p_{K, 3}^{*}(9)=p_{K, 3}(9)=16
$$


since

$$
\begin{aligned}
& \mathfrak{P}_{K, 3}^{*}(9)=\left\{1+8,8+1,1^{2}+7,2+7,1+7+1,1^{4}+5,1^{2}+2+5,\right. \\
& 2^{2}+5,1^{5}+4,1^{3}+2+4,1+2^{2}+4,1+2^{4}, 1^{3}+2^{3}, \\
&\left.1^{5}+2^{2}, 1^{7}+2,1^{9}\right\}
\end{aligned}
$$

and, listed in the corresponding order after the appropriate bijection,

$$
\begin{aligned}
& \mathfrak{P}_{K, 3}(9)=\left\{8+1,5+4,7+1^{2}, 7+2,4^{2}+1,5+1^{4}, 5+2+1^{2},\right. \\
& 5+2^{2}, 4+1^{5}, 4+2+1^{3}, 4+2^{2}+1,2^{4}+1,2^{3}+1^{3}, \\
&\left.2^{2}+1^{5}, 2+1^{7}, 1^{9}\right\} .
\end{aligned}
$$

Another natural question to ask is "How does this bijection affect other classical partition functions?" In what follows, we answer this question with regards to partitions into $h$ colors, and then with regards to partitions into distinct parts.

We now generalize our definitions in a different way, so that we may prove an $h$-colored analog of Theorem 2. A composition of a non-negative integer $n$ into $h$ colors is any sequence of positive integers in which each positive integer is assigned one of $h$ distinct colors, and the sum of all of these positive integers is $n$. A partition of a non-negative integer $n$ into $h$ colors is a non-increasing sequence of positive integers in which each positive integer is assigned one of $h$ distinct colors, the order of the colors is not considered, and the sum of all of these positive integers is $n$. For our purposes, we will always let the set of available colors be $\{1, \ldots, h\}$. Since the order of the colors is not considered, when we are listing parts of the same size, we will list them in non-increasing color order. We will indicate the color of a part with a subscript on the left (e.g., ${ }_{4} 3$ is a part of size 3 and color 4). In situations where we are only concerned with the size of the part being considered, we will suppress the subscript on the left.

Let ${ }_{h} \mathfrak{P}_{k, m}$ be the set of all partitions into $h$ colors where each part is $\equiv k$ $(\bmod m)$, let ${ }_{h} \mathfrak{P}_{k, m}(n)$ be the set of all partitions in ${ }_{h} \mathfrak{P}_{k, m}$ such that the sum of the parts is $n$, let ${ }_{h} p_{k, m}(n)=\left.\right|_{h} \mathfrak{P}_{k, m}(n) \mid$, let ${ }_{h} \mathfrak{P}_{k, m}(j, n)$ be the set of all partitions in ${ }_{h} \mathfrak{P}_{k, m}(n)$ into $j$ parts, and let ${ }_{h} p_{k, m}(j, n)=\left|{ }_{h} \mathfrak{P}_{k, m}(j, n)\right|$.

Definition. Let $1 \leq k \leq m$. A partition into $h$ colors where each part $i s \equiv k(\bmod m)$ with parts of size $k$ in the gaps is any composition into $h$ colors that may be constructed in the following way.

- For $b \geq 0$, let $\bigoplus_{i=1}^{b} c_{i} d_{i} \in{ }_{h} \mathfrak{P}_{k, m}$ be such that $d_{b} \geq m+k, d_{i}-d_{i+1} \geq$ $2 m$ for all $1 \leq i<b$, and if $c_{i}<c_{i+1}$, then $d_{i}-d_{i+1} \neq 2 m$.

- Define $g_{0}=\infty, g_{b}=\left(d_{b}-k\right) / m-1$, and $g_{i}=\left(d_{i}-d_{i+1}\right) / m-2$ for all $1 \leq i<b$. 
- Then

$$
\pi=\bigoplus_{r=1}^{h} k^{e_{r, 0}} \oplus \bigoplus_{i=1}^{b}\left({ }_{c_{i}} d_{i} \bigoplus_{r=1}^{h} k^{e_{r, i}}\right),
$$

where $0 \leq \sum_{r=1}^{h} e_{r, i} \leq g_{i}$ for $i=0,1, \ldots, b$ and $e_{r, b}=0$ if $d_{b}-m \sum_{r=1}^{t} e_{r, b}=$ $m+k$ and $c_{b}<r$, is a partition into $h$ colors where each part is $\equiv k(\bmod m)$ with parts of size $k$ in the gaps.

Let ${ }_{h} \mathfrak{P}_{k, m}^{*}$ be the set of all partitions into $h$ colors where each part is $\equiv k(\bmod m)$ with parts of size $k$ in the gaps, let ${ }_{h} \mathfrak{P}_{k, m}^{*}(n)$ be the set of all partitions in ${ }_{h} \mathfrak{P}_{k, m}^{*}$ such that the sum of the parts is $n$, let ${ }_{h} p_{k, m}^{*}(n)=$ $\left|\mathfrak{P}_{k, m}^{*}(n)\right|$, let ${ }_{h} \mathfrak{P}_{k, m}^{*}(j, n)$ be the set of all partitions in ${ }_{h} \mathfrak{P}_{k, m}^{*}(n)$ with $j$ parts, and let ${ }_{h} p_{k, m}^{*}(j, n)=\left.\right|_{h} \mathfrak{P}_{k, m}^{*}(j, n) \mid$.

We are now prepared to state

THEOREM 4. For all non-negative integers $h, j, k, m$, and $n$ with $1 \leq k \leq m$

$$
{ }_{h} p_{k, m}^{*}(j, n)={ }_{h} p_{k, m}(j, n) .
$$

We find that the proof of Theorem 4 is analogous to the proof of Theorem 1 if we allow the "stolen" parts which we give to the poor to change color as is necessary.

Once again, summing on $j$ yields a meaningful corollary.

Corollary 5. For all non-negative integers $h, k, m$, and $n$ with $1 \leq k$ $\leq m$

EXAMPLE.

$$
{ }_{h} p_{k, m}^{*}(n)={ }_{h} p_{k, m}(n) \text {. }
$$

since

$$
{ }_{2} p_{1,3}^{*}(8)={ }_{2} p_{1,3}(8)=26
$$

$$
\begin{aligned}
&{ }_{2} \mathfrak{P}_{1,3}^{*}(8)=\left\{{ }_{1} 1+{ }_{1} 7,{ }_{2} 1+{ }_{1} 7,{ }_{1} 1+{ }_{2} 7,{ }_{2} 1+{ }_{2} 7,{ }_{1} 7+{ }_{1} 1,{ }_{2} 7+{ }_{1} 1,{ }_{2} 7+{ }_{2} 1,\right. \\
&{ }_{1} 1^{4}+{ }_{1} 4,{ }_{1} 1^{3}+{ }_{2} 1+{ }_{1} 4,{ }_{1} 1^{2}+{ }_{2} 1^{2}+{ }_{1} 4,{ }_{1} 1+{ }_{2} 1^{3}+{ }_{1} 4, \\
&{ }_{2} 1^{4}+{ }_{1} 4,{ }_{1} 1^{4}+{ }_{2} 4,{ }_{1} 1^{3}+{ }_{2} 1+{ }_{2} 4,{ }_{1} 1^{2}+{ }_{2} 1^{2}+{ }_{2} 4, \\
&{ }_{1} 1+{ }_{2} 1^{3}+{ }_{2} 4,{ }_{2} 1^{4}+{ }_{2} 4,{ }_{1} 1^{8},{ }_{1} 1^{7}+{ }_{2} 1,{ }_{1} 1^{6}+{ }_{2} 1^{2},{ }_{1} 1^{5}+{ }_{2} 1^{3}, \\
&\left.{ }_{1} 1^{4}+{ }_{2} 1^{4},{ }_{1} 1^{3}+{ }_{2} 1^{5},{ }_{1} 1^{2}+{ }_{2} 1^{6},{ }_{1} 1+{ }_{2} 1^{7},{ }_{2} 1^{8}\right\}
\end{aligned}
$$

and, listed in the corresponding order after the appropriate bijection,

$$
\begin{aligned}
{ }_{2} \mathfrak{P}_{1,3}(8)=\{ & { }_{1} 7+{ }_{1} 1,{ }_{1} 7+{ }_{2} 1,{ }_{2} 7+{ }_{1} 1,{ }_{2} 7+{ }_{2} 1,{ }_{1} 4^{2},{ }_{2} 4+{ }_{1} 4,{ }_{2} 4^{2},{ }_{1} 4+{ }_{1} 1^{4}, \\
& { }_{1} 4+{ }_{2} 1+{ }_{1} 1^{3},{ }_{1} 4+{ }_{2} 1^{2}+{ }_{1} 1^{2},{ }_{1} 4+{ }_{2} 1^{3}+{ }_{1} 1,{ }_{1} 4+{ }_{2} 1^{4}, \\
& { }_{2} 4+{ }_{1} 1^{4},{ }_{2} 4+{ }_{2} 1+{ }_{1} 1^{3},{ }_{2} 4+{ }_{2} 1^{2}+{ }_{1} 1^{2},{ }_{2} 4+{ }_{2} 1^{3}+{ }_{1} 1 \\
& 2^{4}+{ }_{2} 1^{4},{ }_{1} 1^{8},{ }_{2} 1+{ }_{2} 1^{7},{ }_{2} 1^{2}+{ }_{1} 1^{6},{ }_{2} 1^{3}+{ }_{1} 1^{5},{ }_{2} 1^{4}+{ }_{1} 1^{4} \\
& \left.{ }_{2} 1^{5}+{ }_{1} 1^{3},{ }_{2} 1^{6}+{ }_{1} 1^{2},{ }_{2} 1^{7}+{ }_{1} 1,{ }_{2} 1^{8}\right\} .
\end{aligned}
$$


Finally, the method of Theorem 1 can also be used to give bijections between partitions with parts in the gaps and ordinary partitions into distinct parts. The key to doing this is to modify the definitions of the gapspaces and to replace the minimal difference $2 m$ conditon with a minimal difference $3 m$ condition. We will call these new partitions with parts in the gaps "smallgap partitions".

Let $\mathfrak{Q}_{k, m}$ be the set of all partitions into distinct parts where each part is $\equiv k(\bmod m)$, let $\mathfrak{Q}_{k, m}(n)$ be the set of all partitions of $n$ into distinct parts where each part is $\equiv k(\bmod m)$, let $q_{k, m}(n)=\left|\mathfrak{Q}_{k, m}(n)\right|$, let $\mathfrak{Q}_{k, m}(j, n)$ be the set of all partitions of $n$ into $j$ distinct parts where each part is $\equiv k$ $(\bmod m)$, and let $q_{k, m}(j, n)=\left|\mathfrak{Q}_{k, m}(j, n)\right|$.

Definition. Let $1 \leq k \leq m$. A smallgap partition where each part is $\equiv k(\bmod m)$ with parts of size $k$ in the gaps is any composition that may be constructed in the following way:

- For $b \geq 0$, let $\bigoplus_{i=1}^{b} d_{i} \in \mathfrak{Q}_{k, m}$ be such that $d_{b} \geq 2 m+k$ and $d_{i}-d_{i+1} \geq$ $3 m$ for all $1 \leq i<b$.

- Define $g_{0}=\infty$,

$$
\begin{aligned}
g_{b} & = \begin{cases}1 & \text { if } d_{b}>2 m+k, \\
0 & \text { if } d_{b}=2 m+k,\end{cases} \\
g_{i} & =\left\{\begin{array}{ll}
1 & \text { if } d_{i}-d_{i+1}>3 m, \\
0 & \text { if } d_{i}-d_{i+1}=3 m,
\end{array} \text { for all } 1 \leq i<b .\right.
\end{aligned}
$$

- Then

$$
\pi=k^{e_{0}} \oplus \bigoplus_{i=1}^{b}\left(d_{i} \oplus k^{e_{i}}\right)
$$

where $0 \leq e_{i} \leq g_{i}$ for $i=0,1, \ldots, b$ is a smallgap partition where each part is $\equiv k(\bmod m)$ with parts of size $k$ in the gaps.

Let $\mathfrak{Q}_{k, m}^{*}$ be the set of all smallgap partitions where each part is $\equiv k$ $(\bmod m)$ with parts of size $k$ in the gaps, let $\mathfrak{Q}_{k, m}^{*}(n)$ be the set of all smallgap partitions in $\mathfrak{Q}_{k, m}^{*}$ such that the sum of the parts is $n$, let $q_{k, m}^{*}(n)=$ $\left|\mathfrak{Q}_{k, m}^{*}(n)\right|$, let $\mathfrak{Q}_{k, m}^{*}(j, n)$ be the set of all smallgap partitions in $\mathfrak{Q}_{k, m}^{*}(n)$ with $j$ parts, and let $q_{k, m}^{*}(j, n)=\left|\mathfrak{Q}_{k, m}^{*}(j, n)\right|$.

We are now prepared to state

TheOrem 5. For all non-negative integers $j, k, m$, and $n$ with $1 \leq k$ $\leq m$,

$$
q_{k, m}^{*}(j, n)=q_{k, m}(j, n) .
$$

We find that we can either prove Theorem 5 directly in a manner analogous to the way we proved Theorem 1 , or we can prove the $k=m=1$ case 
first, and then show that Theorem 5 follows from that case in the same way that Theorem 2 followed from Theorem 1.

As is always the case, summing on $j$ yields a meaningful corollary.

COROLlaRY 6. For all non-negative integers $k, m$, and $n$ with $1 \leq k \leq m$,

EXAMPLE.

$$
q_{k, m}^{*}(n)=q_{k, m}(n) .
$$

since

$$
q_{3,4}^{*}(90)=q_{3,4}(90)=14
$$

$$
\begin{aligned}
& \mathfrak{Q}_{3,4}^{*}(90)=\{3+87,87+3,83+7,79+11 \\
& 75+15,71+19,67+23,63+27,59+31, \\
& 55+35,51+39,3+51+3+23+3+7, \\
&3+47+3+27+3+7,3+43+3+27+3+11\}
\end{aligned}
$$

and, listed in the corresponding order after the appropriate bijection,

$$
\begin{aligned}
& \mathfrak{Q}_{3,4}(90)=\{87+3,83+7,79+11,75+15,71+19, \\
& 67+23,63+27,59+31,55+35,51+39, \\
& 47+43,35+19+15+11+7+3, \\
&31+23+15+11+7+3,27+23+19+11+7+3\} .
\end{aligned}
$$

If we wished, we could create many new results by taking combinations of Theorems 3, 4, and 5, but stating such theorems is quite cumbersome. For that reason, we will forego the statement of such theorems here.

5. Conclusion. We have now seen how the notion of stealing from the rich and giving to the poor gives rise to many natural generalizations of some results in [4]. It may be of interest to further explore how this type of bijection can be extended to produce identities between other types of partitions. Furthermore, it has recently come to light that this bijection can actually be used to demonstrate the equivalence of some of the results of Alladi and Bowman; this link will be the subject of a future project by the author.

\section{References}

[1] K. Alladi, Partition identities involving gaps and weights, Trans. Amer. Math. Soc. 349 (1997), 5001-5019.

[2] -, Partition identities involving gaps and weights - II, Ramanujan J., to appear.

[3] - Weighted partition identities and applications, in: Analytic Number Theory, Proceedings of a Conference in Honor of Heini Halberstam, Vol. 1 (Allerton Park, Ill., 1995), Progr. Math. 138, Birkhäuser, Boston, 1996, 1-15. 
[4] D. Bowman, Partitions with numbers in their gaps, Acta Arith. 74 (1996), 97-105.

[5] L. Euler, Introductio in Analysis Infinitorum, Marcum-Michaelem Bousquet, Lousannae, 1748.

Department of Mathematics

The University of Illinois

1409 West Green Street

Urbana, Illinois 61801-2975

U.S.A.

E-mail: eichhorn@math.uiuc.edu

Received on 26.11.1996

and in revised form on 26.9.1997 